\title{
Differences in potential biomarkers of delirium between acutely ill medical and elective cardiac surgery patients
}

This article was published in the following Dove Medical Press journal:

Clinical Interventions in Aging

\author{
Angelique Egberts' \\ Robert J Osse ${ }^{2}$ \\ Durk Fekkes ${ }^{2,3}$ \\ Joke HM Tulen ${ }^{2}$ \\ Tischa JM van der Cammen' \\ Francesco US \\ Mattace-Raso' \\ 'Section of Geriatric Medicine, \\ Department of Internal Medicine, \\ Erasmus MC University Medical \\ Center, Rotterdam, the Netherlands; \\ ${ }^{2}$ Department of Psychiatry, Erasmus \\ MC University Medical Center, \\ Rotterdam, the Netherlands; \\ ${ }^{3}$ Department of Clinical Chemistry, \\ Erasmus MC University Medical \\ Center, Rotterdam, the Netherlands
}

Correspondence: Angelique Egberts; Francesco US Mattace-Raso Section of Geriatric Medicine, Department of Internal Medicine, Erasmus MC University Medical Center, Room Rg 527, PO Box 2040, 3000 CA Rotterdam, the Netherlands $\mathrm{Tel}+3$ I I0 7035979 Fax +3I 107034768 Email a.egberts@erasmusmc.nl; f.mattaceraso@erasmusmc.nl
Background/aims: The pathophysiology of delirium is poorly understood. Increasing evidence suggests that different pathways might be involved in the pathophysiology depending on the population studied. The aim of the present study was to investigate potential differences in mean plasma levels of neopterin, amino acids, amino acid ratios and homovanillic acid between two groups of patients with delirium.

Methods: Data from acutely ill medical patients aged 65 years and older, and patients aged 70 years and older undergoing elective cardiac surgery, were used. Differences in biomarker levels between the groups were investigated using univariate ANOVA with adjustments for age, sex, comorbidities, C-reactive protein (CRP) and the estimated glomerular filtration rate (eGFR), where appropriate. Linear regression analysis was used to identify potential determinants of the investigated biochemical markers.

Results: Eighty patients with delirium were included (23 acutely ill medical patients and 57 elective cardiac surgery patients). After adjustment, higher mean neopterin levels (93.1 vs $47.3 \mathrm{nmol} / \mathrm{L}, P=0.001)$ and higher phenylalanine/tyrosine ratios ( 1.39 vs $1.15, P=0.032)$ were found in acutely ill medical patients when compared to elective cardiac surgery patients. CRP levels were positively correlated with neopterin levels in acutely ill medical patients, explaining $28.4 \%$ of the variance in neopterin levels. eGFR was negatively correlated with neopterin in elective cardiac surgery patients, explaining $53.7 \%$ of the variance in neopterin levels.

Conclusion: In this study, we found differences in mean neopterin levels and phenylalanine/ tyrosine ratios between acutely ill medical and elective cardiac surgery patients with delirium. Moreover, our findings may suggest that in acutely ill medical patients, neopterin levels are mainly determined by inflammation/oxidative stress whereas in elective cardiac surgery patients, neopterin levels are mainly driven by renal function/fluid status. These findings suggest that the markers and pathways that might be involved in the pathophysiology of delirium may differ between specific groups of patients.

Keywords: delirium, inflammation, neopterin, oxidative stress, renal function

\section{Introduction}

Delirium - an acute neuropsychiatric syndrome characterized by disturbances in attention, awareness and cognition - is a frequent disorder in older hospitalized patients. ${ }^{1-3}$ It is associated with poor clinical outcomes including prolonged hospital stay, loss of independence, increased risk of cognitive decline and mortality. ${ }^{4}$ Although it is widely accepted that the cause of delirium is multifactorial with a complex interplay between predisposing factors (eg, advanced age and dementia) and precipitating factors (eg, acute medical illness and surgery), the pathophysiology of delirium is still poorly understood. ${ }^{3}$ 
Increasing evidence suggests that delirium might have different pathophysiological mechanisms depending on the precipitating factor and the health status of the patient (acutely ill or relatively healthy). ${ }^{5,6}$ Several mechanisms may play a role and include, among others, activation of the immune system, oxidative stress and disturbances in serotonergic and dopaminergic neurotransmission. ${ }^{7}$ During immune system activation, monocytes and macrophages are stimulated to produce neopterin. ${ }^{8}$ The association between this novel potential biomarker, neopterin, and delirium has been investigated in three previous studies which included acutely ill medical patients, ${ }^{9}$ elective patients undergoing cardiac surgery ${ }^{10}$ and acute hip fracture patients undergoing surgery. ${ }^{11}$ In all three studies, neopterin levels were elevated in patients with delirium. However, when comparing the results of the studies, large differences are observed in mean neopterin levels between the different studies. Considerably lower mean neopterin levels were found in surgical patients with delirium ${ }^{10,11}$ than in acutely ill medical patients with delirium. ${ }^{9}$ Also interestingly, neopterin levels measured in patients with delirium after cardiac surgery ${ }^{10}$ were in the same order as neopterin levels measured in acutely ill medical patients without delirium. ${ }^{9}$

Disturbances in serotonergic and dopaminergic neurotransmission may also contribute to delirium. ${ }^{7}$ The synthesis of serotonin in the central nervous system is, among others, dependent on the availability of its precursor, the large neutral amino acid (LNAA) tryptophan. Tryptophan competes with the other LNAAs (phenylalanine, tyrosine, valine, leucine and isoleucine) for transport across the blood-brain barrier. The amount of tryptophan that eventually enters the brain is determined by the ratio of tryptophan to the sum of the other LNAAs. ${ }^{12}$ Therefore, a reduced ratio may result in a decreased synthesis of serotonin. Several studies have found decreased levels of tryptophan and tryptophan/LNAAs ratios in patients with delirium, ${ }^{10,12-15}$ whereas other studies did not report differences in these parameters between patients with and without delirium. ${ }^{16-18}$

It has also been suggested that an increased dopaminergic neurotransmission plays a role in delirium. The amino acids phenylalanine and tyrosine are the precursors of dopamine and the ratios of phenylalanine and tyrosine to the other LNAAs are suggestive of the amount of phenylalanine and tyrosine that enters the brain. In patients with delirium, increased ratios, ${ }^{17}$ as well as increased plasma levels of dopamine's metabolite homovanillic acid (HVA), have been found, ${ }^{10,16}$ but the results are inconclusive. ${ }^{19}$

In addition, serotonin and dopamine are synthesized from tryptophan, phenylalanine and tyrosine in enzymatic reactions that use tetrahydrobiopterin $\left(\mathrm{BH}_{4}\right)$ as an essential cofactor. ${ }^{20} \mathrm{~A}$ change in the availability of $\mathrm{BH}_{4}$ may therefore affect the synthesis of these neurotransmitters. The ratio of phenylalanine to tyrosine (Phe/Tyr ratio) can be used as an indirect measure of $\mathrm{BH}_{4}$. This ratio reflects the activity of the peripherally located enzyme phenylalanine hydroxylase, an enzyme that requires $\mathrm{BH}_{4}$ to convert phenylalanine into tyrosine. ${ }^{20,21} \mathrm{An}$ increased Phe/Tyr ratio suggests a reduced availability of $\mathrm{BH}_{4}$. In a previous study, an increased ratio was found in patients with delirium, ${ }^{19}$ whereas another study reported no difference between patients with and without delirium. ${ }^{10}$

These controversial findings can be caused by differences in analytical procedures but can also be suggestive of potential differences in biochemical profiles and pathophysiological pathways between patient groups with delirium. Adequate knowledge regarding possible differences in the pathophysiology is required to improve delirium prevention and treatment.

The aim of this exploratory study was to investigate potential differences in mean plasma levels of neopterin, amino acids, amino acid ratios and HVA between two specific groups of patients with delirium, ie, acutely ill medical patients with delirium and patients with a delirium after elective cardiac surgery.

\section{Methods \\ Study populations}

In this study, we used data from the Delirium In The Old (DITO) study ${ }^{9,19}$ and the DElirium pathogenesis, Cognition and Outcome (DECO) study. ${ }^{10}$ In both studies, mean levels of neopterin, several amino acids, amino acid ratios and HVA in patients with and without delirium were compared and the results can be found elsewhere. ${ }^{9,10,19}$ Only patients with delirium were included in the present study. The most relevant procedures of the two studies will be described here; detailed information can be found in previous publications. ${ }^{9,10,19}$

The DITO study was a cross-sectional study in patients aged 65 years and older who were acutely ill and admitted to the wards of Internal Medicine and Geriatrics of the Erasmus University Medical Center (Erasmus MC) and the ward of Geriatrics of the Harbour Hospital, Rotterdam, the Netherlands. Exclusion criteria were a diagnosis of Lewy body dementia, Parkinson's disease, neuroleptic malignant syndrome, tardive dyskinesia, ongoing treatment with antipsychotics or other psychiatric medications except haloperidol and benzodiazepines, aphasia, insufficient understanding of the Dutch language and a Mini-Mental State Examination (MMSE) score $<10$ points out of 30 . 
The DECO study was a prospective cohort study in patients aged 70 years and older who underwent elective cardiac surgery (coronary artery bypass graft [CABG], valve surgery or both) at the Department of Cardiothoracic Surgery of the Erasmus MC. Exclusion criteria were surgery in which deep hypothermia circulatory arrest or an emergency procedure was required, insufficient understanding of the Dutch language, preoperative delirium and insufficient adherence to the protocol.

In both studies, written informed consent was obtained from all participants or their representatives. The study protocols were approved by the Medical Ethics Committee of the Erasmus MC. All methods were performed in accordance with the relevant guidelines and regulations.

\section{Procedures}

In both studies, participants were assessed daily for the presence of delirium by the nursing and medical staff and by members of the research teams until discharge or until 7 days after surgery.

In the DITO study, the 13-items Delirium Observation Screening scale was used to screen for a change in behavior. ${ }^{22}$ The diagnosis of delirium was made by a geriatrician, according to the criteria of the Diagnostic and Statistical Manual of Mental Disorders, fourth edition (DSM-IV), ${ }^{1}$ and was based on psychiatric examination of the patient, the medical and nursing records, the Delirium Observation Screening scale scores and information given by the patient's closest relative. Cognitive functioning was assessed in the absence of delirium, at a later moment during the hospital stay, using the MMSE. ${ }^{23}$ When it was impossible to score the MMSE during admission because the patient was too ill, the cognitive functioning was discussed with a clinician or assessed with information from the available medical records. When the clinical opinion was that the patient would have a MMSE score $\geq 10$, the patient was not excluded from the study.

In the DECO study, the diagnosis of delirium was made by a senior psychiatrist and/or a trained researcher in daily assessments between 10:00 and 12:00 hours using the Confusion Assessment Method for the Intensive Care Unit (which follows the criteria for delirium of the DSM-IV) ${ }^{24}$ and was based on psychiatric examination of the patient and information in the medical and nursing records. Delirium lasting $>1$ day was considered clinically relevant. The first day after surgery was not taken into account because of possible residual effects of anesthesia. On the day before surgery, cognitive functioning was assessed using the MMSE.

In the DITO study, blood samples were collected within 48 hours after admission. When a patient developed delirium during the hospital stay, new blood samples were collected within 24 hours after the onset of delirium and were used instead of the first blood samples for the statistical analyses. In the DECO study, blood samples were collected on the day before surgery and the second day after surgery.

In the present study, we compared the biochemical data collected in the DITO study with the postoperative biochemical data from the DECO study.

\section{Biochemical measurements}

In both studies, non-fasting blood was collected preferably between 8 and $10 \mathrm{am}$ in an 8 - $\mathrm{mL}$ tube containing ethylene diamine tetra-acetic acid. After blood sampling, the tubes were protected from light to prevent oxidative loss of neopterin ${ }^{25}$ and stored at room temperature to prevent changes in the transfer of amino acids between plasma and blood cells. ${ }^{26}$ Within 3 hours, the blood was centrifuged for 20 minutes at $2,650 \mathrm{~g}$ and $20^{\circ} \mathrm{C}$. The obtained plasma was stored at $-80^{\circ} \mathrm{C}$ until analysis.

In both studies, neopterin, amino acids and HVA were determined by the same procedures and by the same analytical staff. Plasma neopterin levels were determined by HPLC after acid oxidation. ${ }^{21}$ Plasma amino acid levels were measured using HPLC with automated pre-column derivatization with ortho-phthalaldehyde, ${ }^{26}$ and plasma HVA levels were determined by reversed-phase HPLC and electrochemical detection, as previously described for the measurement of serotonin. ${ }^{27}$

C-reactive protein (CRP) levels and the estimated glomerular filtration rate (eGFR) were taken from the medical records. In case of the DECO study, the 2-day postoperative levels were recorded. The eGFR was determined by the following Modification of Diet in Renal Disease formula: $175 \times[\text { serum creatinine }(\mu \mathrm{mol} / \mathrm{L}) \times 0.0113]^{-1.154} \times \mathrm{age}^{-0.203} \times$ 0.742 (if female).

\section{Statistical analysis}

Differences in participant characteristics between acutely ill medical and elective cardiac surgery patients with delirium were evaluated using the chi-squared test or Fisher's exact test for categorical variables and the Mann-Whitney $U$-test or the Student's $t$-test for continuous variables, depending on the distribution of the data.

Univariate ANOVA was used to investigate potential differences in mean levels of biochemical parameters (dependent variable) between acutely ill medical and elective cardiac surgery patients. For this purpose, biochemical parameters that were not normally distributed were logarithmically transformed (neopterin, HVA, all amino acids and amino 
acid ratios). Analyses were adjusted for age, sex and Charlson Comorbidity Index (CCI). The model including neopterin was additionally adjusted for CRP and eGFR, since neopterin is an inflammatory marker which is mainly excreted by the kidneys. ${ }^{8}$ In additional analyses, neopterin, HVA, all amino acids and amino acid ratios were also adjusted for MMSE score. All mean levels and corresponding 95\% CIs presented in this manuscript are the back-transformed $\log _{10}$-values.

Unadjusted linear regression analysis was performed to identify potential determinants of biochemical parameters, which were statistically significantly different between the two groups. Second, variables with a $P$-value $\leq 0.10$ were included in a multiple linear regression analysis and stratified for study, to determine the relative contribution of each variable to the total variance in the biochemical parameter. Semi-partial (part) correlation coefficients were squared to calculate the percentage of total variance that was explained by each variable.
Due to the exploratory nature of this study, no correction for multiple comparisons was applied. All statistical analyses were performed using SPSS, version 21.0 (IBM Co., Armonk, NY, USA). Results were considered statistically significant at a two-sided $P<0.05$. Figures were constructed using GraphPad Prism 5.01 for Windows (GraphPad Software, San Diego, CA, USA).

\section{Results}

\section{Participant characteristics}

Of the 211 patients enrolled in the two studies, 81 were diagnosed with delirium. One participant from the DECO study was excluded due to withdrawal of consent. In total, 80 patients with delirium were included, 23 acutely ill medical patients and 57 elective cardiac surgery patients. Of the 23 acutely ill medical patients with delirium, 21 had delirium on admission and 2 developed delirium during the hospital stay. Table 1 presents the demographic and clinical characteristics

Table I Characteristics of the study participants

\begin{tabular}{|c|c|c|c|}
\hline & DITO (n=23) & DECO $(n=57)$ & $P$-value \\
\hline Male, n (\%) & $10(43.5)$ & $34(59.6)$ & $0.188^{\mathrm{a}}$ \\
\hline Age, years, mean \pm SD & $85.9 \pm 4.0$ & $76.8 \pm 3.8$ & $<0.001^{b}$ \\
\hline MMSE score, median (IQR) $)^{c, d}$ & $20.0(18.0-25.0)^{\mathrm{e}}$ & $27.0(24.0-28.0)$ & $<0.00 I^{f}$ \\
\hline CCl score, median (IQR) & $2.0(1.0-3.0)$ & $2.0(1.0-3.0)$ & $0.550^{f}$ \\
\hline \multicolumn{4}{|l|}{$\mathrm{CCl}$ score, n (\%) } \\
\hline 0 & $\mathrm{I}(4.3)$ & II (19.3) & \\
\hline $\mathrm{I}$ & $8(34.8)$ & $12(21.1)$ & \\
\hline 2 & $7(30.4)$ & $15(26.3)$ & \\
\hline$\geq 3$ & $7(30.4)$ & $19(33.3)$ & \\
\hline CRP, mg/L, median (IQR) ${ }^{g}$ & $36.0(10.0-103.0)$ & $100.0(60.0-148.0)$ & $0.013^{f}$ \\
\hline eGFR, $\mathrm{mL} / \mathrm{min}$, mean $\pm \mathrm{SD}^{\mathrm{g}}$ & $49.4 \pm 25.1$ & $48.9 \pm 23.2$ & $0.930^{\mathrm{b}}$ \\
\hline Acute kidney injury, $\mathrm{n}(\%)^{\mathrm{h}}$ & $2(8.7)$ & $8(14.0)$ & $0.716^{i}$ \\
\hline \multicolumn{4}{|l|}{ Reasons for admission, $\mathrm{n}(\%)^{\mathrm{j}}$} \\
\hline Infection & $17(73.9)$ & - & \\
\hline Electrolyte disorder & $5(21.7)$ & - & \\
\hline Dehydration & $4(17.4)$ & - & \\
\hline Heart failure & $2(8.7)$ & - & \\
\hline Carcinoma & $2(8.7)$ & - & \\
\hline Urinary retention & $2(8.7)$ & - & \\
\hline Atrial fibrillation & I (4.3) & - & \\
\hline Adverse drug event & $\mathrm{I}(4.3)$ & - & \\
\hline Multiple organ dysfunction & $\mathrm{I}(4.3)$ & - & \\
\hline Fever of unknown origin & $\mathrm{I}(4.3)$ & - & \\
\hline Elective cardiac surgery & - & 57 (100\%) & \\
\hline
\end{tabular}

Notes: Values are expressed as mean \pm SD for normally distributed continuous variables, median (IQR) for not normally distributed continuous variables and $\mathrm{n}$ (percentages)

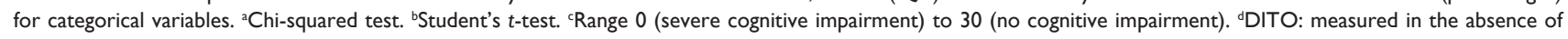
delirium, DECO: measured on the day before surgery. ${ }^{e} F o u r$ values missing. 'Mann-Whitney U-test. ${ }^{8} \mathrm{DITO}$ : measured during delirium (on the day of neopterin sampling), DECO: measured during delirium 2 days after cardiac surgery. "Defined as a decrease in eGFR of $>50 \%$ in comparison to baseline or the preoperative situation. 'Fisher's exact test. Percentages do not add up to $100 \%$ due to multiple admission reasons per patient.

Abbreviations: CCI, Charlson Comorbidity Index; CRP, C-reactive protein; DITO, Delirium In The Old; DECO, DElirium pathogenesis, Cognition and Outcome; eGFR, estimated glomerular filtration rate; IQR, interquartile range; MMSE, Mini-Mental State Examination. 
of the participants. Acutely ill medical patients with delirium were older (mean 85.9 vs 76.8 years, $P<0.001$ ), had lower MMSE scores (median 20 vs $27, P<0.001$ ) and lower CRP levels (median 36.0 vs $100.0 \mathrm{mg} / \mathrm{L}, P=0.013$ ) than elective cardiac surgery patients with delirium.

\section{Analyses of biochemical parameters}

The adjusted mean levels and corresponding 95\% CIs of the investigated biochemical parameters in acutely ill medical and elective cardiac surgery patients are presented in Table 2. After adjustment for age, sex, CCI, eGFR and CRP levels, mean neopterin levels were significantly higher in acutely ill medical patients ( $93.1 \mathrm{nmol} / \mathrm{L}, 95 \%$ CI: 69.3-125.3) than in elective cardiac surgery patients $(47.3 \mathrm{nmol} / \mathrm{L}, 95 \% \mathrm{CI}$ : 40.1-55.8) ( $P=0.001)$. Furthermore, mean Phe/Tyr ratios were significantly higher in acutely ill medical patients $(1.39$, 95\% CI: $1.22-1.58)$ than in elective cardiac surgery patients $(1.15,95 \%$ CI: $1.07-1.24)(P=0.032)$. No statistically significant differences were found in the mean levels of the other biochemical parameters. Estimates remained unchanged after additional adjustment for MMSE scores (Table S1).

\section{Determinants of neopterin}

Unadjusted linear regression analysis revealed that neopterin levels were positively correlated with CRP levels in acutely ill medical patients with delirium and negatively correlated with eGFR in elective cardiac surgery patients with delirium (Table 3, Figure 1). Trends toward lower neopterin levels were found for increasing age $(r=-0.399, P=0.060)$ and increasing eGFR $(r=-0.396, P=0.061)$ in acutely ill medical patients (Table 3, Figure 1). No correlation was found between neopterin, CCI and MMSE scores in both groups of patients $(P>0.10)$ (Table 3$)$.

Multiple linear regression analysis revealed that a model based on age, CRP and eGFR could explain $53.2 \%$ of the variance in neopterin levels in acutely ill medical patients with delirium (Table 4). In this model, CRP levels remained positively correlated with neopterin levels and explained $28.4 \%$ of the variance in neopterin levels. Age and eGFR explained 8.53\% and $3.96 \%$, respectively of the variance in neopterin levels, but were not statistically significant determinants of neopterin. In elective cardiac surgery patients with delirium, the same model explained $56.7 \%$ of the variance in neopterin levels (Table 4 ).

Table 2 Adjusted mean levels of biochemical parameters

\begin{tabular}{|c|c|c|c|}
\hline & DITO $(n=23)$ & $\operatorname{DECO}(n=57)$ & $P$-value \\
\hline Neopterin, nmol/L & $84.1(57.0-123.9)$ & $49.4(40.0-61.0)$ & 0.038 \\
\hline Neopterin, nmol/ $\mathrm{L}^{\mathrm{a}}$ & $93.1(69.3-125.3)$ & $47.3(40.1-55.8)$ & 0.001 \\
\hline Homovanillic acid, nmol/L & $120.2(79.6-181.1)^{b}$ & $160.3(128.5-199.5)^{\mathrm{b}}$ & 0.279 \\
\hline Glutamic acid, $\mu \mathrm{mol} / \mathrm{L}$ & $46.7(37.3-58.3)$ & $35.4(31.3-39.9)$ & 0.059 \\
\hline Serine, $\mu \mathrm{mol} / \mathrm{L}$ & $80.2(68.4-94.0)$ & $75.5(69.2-82.2)$ & 0.555 \\
\hline Glycine, $\mu \mathrm{mol} / \mathrm{L}$ & $182.0(\mid 57.0-210.9)$ & $198.2(|82.8-2| 4.3)$ & 0.375 \\
\hline Citrulline, $\mu \mathrm{mol} / \mathrm{L}$ & $24.0(19.3-29.8)$ & $23.1(20.6-26.0)$ & 0.803 \\
\hline Arginine, $\mu \mathrm{mol} / \mathrm{L}$ & $35.4(28.1-44.6)$ & $44.0(38.7-49.9)$ & 0.152 \\
\hline Taurine, $\mu \mathrm{mol} / \mathrm{L}$ & $35.7(27.2-47.1)$ & $33.6(28.9-38.9)$ & 0.722 \\
\hline Tyrosine, $\mu \mathrm{mol} / \mathrm{L}$ & $56.6(47.2-67.9)$ & $66.2(60.0-73.1)$ & 0.189 \\
\hline Valine, $\mu \mathrm{mol} / \mathrm{L}$ & 208.4 (I82.8-237.7) & $202.8(188.8-2 \mid 7.8)$ & 0.758 \\
\hline Methionine, $\mu \mathrm{mol} / \mathrm{L}$ & $22.4(\mid 8.4-27.3)$ & $28.5(25.6-31.8)$ & 0.063 \\
\hline Tryptophan, $\mu \mathrm{mol} / \mathrm{L}^{\mathrm{c}}$ & $30.4(23.5-37.2)$ & $33.8(30.1-37.5)$ & 0.440 \\
\hline Phenylalanine, $\mu \mathrm{mol} / \mathrm{L}$ & $78.5(70.0-88.3)$ & $76.0(7 \mid .4-8 I .1)$ & 0.665 \\
\hline Isoleucine, $\mu \mathrm{mol} / \mathrm{L}$ & $55.8(47.6-65.5)$ & $67.6(61.9-73.6)$ & 0.069 \\
\hline Leucine, $\mu \mathrm{mol} / \mathrm{L}$ & $121.9(104.5-142.2)$ & $123.9(1 \mid 3.8-134.6)$ & 0.875 \\
\hline Phenylalanine/tyrosine ratio & $1.39(1.22-1.58)$ & $1.15(1.07-1.24)$ & 0.032 \\
\hline Tryptophan/LNAAs ratio $\times 100^{\circ}$ & $5.35(4.37-6.32)$ & $6.11(5.59-6.64)$ & 0.228 \\
\hline Tyrosine/LNAAs ratio $\times 100$ & $11.4(9.8-13.2)$ & I3.I (I2.I-|4.2) & 0.152 \\
\hline Phenylalanine/LNAAs ratio $\times 100$ & $16.5(14.6-18.6)$ & $15.3(14.4-16.4)$ & 0.363 \\
\hline Citrulline/arginine ratio & $0.68(0.52-0.89)$ & $0.53(0.45-0.61)$ & 0.153 \\
\hline
\end{tabular}

Notes: Values are expressed as mean $(95 \% \mathrm{Cl})$ and are the back-transformed log ${ }_{10}$ values unless otherwise specified. Models are adjusted for age, sex and $\mathrm{CCl}$. ${ }^{\mathrm{A}} \mathrm{Additionally}$ adjusted for the eGFR and CRP. Analysis was performed in 76 patients with delirium (DITO $n=23$ and DECO $n=53$ ) due to missing CRP levels. ${ }^{b}$ One value missing. ${ }^{~} V$ alues were not logarithmically transformed.

Abbreviations: CCl, Charlson Comorbidity Index; CRP, C-reactive protein; DITO, Delirium In The Old; DECO, DElirium pathogenesis, Cognition and Outcome; eGFR, estimated glomerular filtration rate; LNAAs, large neutral amino acids. 
Table 3 Unadjusted linear regression analyses of potential determinants of neopterin and Phe/Tyr ratio

\begin{tabular}{|c|c|c|c|c|c|c|c|c|}
\hline \multirow[t]{2}{*}{ Variable } & \multicolumn{4}{|c|}{$\log _{10}($ neopterin $[\mathrm{nmol} / \mathrm{L}])$ in DITO } & \multicolumn{4}{|c|}{$\log _{10}($ neopterin $[\mathrm{nmol} / \mathrm{L}])$ in DECO } \\
\hline & B & $95 \% \mathrm{Cl}$ & $P$-value & $R^{2}$ & B & $95 \% \mathrm{Cl}$ & $P$-value & $R^{2}$ \\
\hline Age & -0.032 & $(-0.064 ; 0.001)$ & 0.060 & 0.159 & 0.006 & $(-0.015 ; 0.026)$ & 0.583 & 0.006 \\
\hline Sex & -0.085 & $(-0.370 ; 0.199)$ & 0.539 & 0.018 & -0.018 & $(-0.176 ; 0.139)$ & 0.816 & 0.001 \\
\hline $\mathrm{CCl}$ & 0.039 & $(-0.043 ; 0.122)$ & 0.332 & 0.045 & 0.022 & $(-0.024 ; 0.069)$ & 0.339 & 0.017 \\
\hline CRP & 0.002 & $(0.00 I ; 0.004)$ & 0.002 & 0.364 & 0.001 & $(0.000 ; 0.002)$ & 0.221 & 0.029 \\
\hline eGFR & -0.005 & $(-0.010 ; 0.000)$ & 0.061 & 0.157 & -0.009 & $(-0.011 ;-0.007)$ & $<0.001$ & 0.537 \\
\hline \multirow[t]{2}{*}{ MMSE } & 0.001 & $(-0.031 ; 0.034)$ & 0.940 & 0.000 & 0.004 & $(-0.023 ; 0.03 I)$ & 0.756 & 0.002 \\
\hline & \multicolumn{4}{|c|}{$\log _{10}$ (Phe/Tyr ratio) in DITO } & \multicolumn{4}{|c|}{$\log _{10}$ (Phe/Tyr ratio) in DECO } \\
\hline Age & -0.006 & $(-0.021 ; 0.009)$ & 0.436 & 0.029 & -0.001 & $(-0.007 ; 0.005)$ & 0.765 & 0.002 \\
\hline Sex & 0.049 & $(-0.072 ; 0.170)$ & 0.411 & 0.032 & -0.010 & $(-0.056 ; 0.035)$ & 0.647 & 0.004 \\
\hline $\mathrm{CCl}$ & 0.023 & $(-0.012 ; 0.057)$ & 0.189 & $0.08 I$ & 0.003 & $(-0.011 ; 0.016)$ & 0.705 & 0.003 \\
\hline MMSE & 0.000 & $(-0.008 ; 0.008)$ & 0.988 & 0.000 & 0.000 & $(-0.0 \mid 4 ; 0.014)$ & 0.966 & 0.000 \\
\hline
\end{tabular}

Notes: Age was measured in years, sex was coded as $\mathrm{I}=$ male, $2=$ female, $\mathrm{CCl}$ was measured in points, CRP was measured in $\mathrm{mg} / \mathrm{L}$, eGFR was measured in $\mathrm{mL} / \mathrm{min}$ and MMSE was measured in points. $B$, regression coefficient; $R^{2}$, squared correlation coefficient.

Abbreviations: $\mathrm{CCl}$, Charlson Comorbidity Index; CRP, C-reactive protein; DITO, Delirium In The Old; DECO, DElirium pathogenesis, Cognition and Outcome; eGFR, estimated glomerular filtration rate; MMSE, Mini-Mental State Examination; Phe, phenylalanine; Tyr, tyrosine.

In this model, eGFR remained negatively correlated with neopterin levels and explained $53.7 \%$ of the variance in neopterin levels. Age and CRP explained $0.18 \%$ and $2.76 \%$ of the variance in neopterin levels, respectively, and were not statistically significant determinants of neopterin levels.

\section{Determinants of Phe/Tyr ratio}

Unadjusted linear regression analysis revealed that age, sex, CCI and MMSE scores were not statistically significantly correlated with Phe/Tyr ratios in both acutely ill medical and elective cardiac surgery patients with delirium (Table 3 ). Also no trends toward correlation were found.

\section{Discussion}

In this study, we found higher mean levels of neopterin and higher Phe/Tyr ratios in acutely ill medical patients with delirium than in patients who developed a delirium after elective cardiac surgery. These findings suggest that the markers and pathways that might be involved in the pathophysiology of delirium in acutely ill medical patients may differ from those in elective cardiac surgery patients.

Delirium frequently occurs in conditions in which the immune system is activated, and this suggests that inflammatory mediators may play a role in the pathophysiology. Several cytokines and inflammatory markers have already been found to be increased in acutely ill medical ${ }^{9,28}$ and cardiac surgery patients ${ }^{29}$ with delirium. Neopterin can be considered as a marker of cell-mediated immune system activation and oxidative stress as it is primarily produced by activated monocytes and macrophages upon stimulation with the pro-inflammatory cytokine interferon-gamma (IFN- $\gamma$ ). Previous studies, including the DITO and DECO study, have found increased neopterin levels in plasma and cerebrospinal fluid prior to and during delirium ${ }^{9-11}$ and this consistency in findings suggests that neopterin might indeed play a role in the pathophysiology of delirium. Nevertheless, in the present study we found a large difference in mean neopterin levels between acutely ill medical and elective cardiac surgery patients with delirium, where all neopterin measurements were performed in the same laboratory using identical procedures. This finding remained significant after adjustment for potential confounders.

Substantial differences in determinants of neopterin levels were found between the two groups of patients with delirium. CRP levels were the strongest determinant of neopterin levels in acutely ill medical patients with delirium, explaining $28.4 \%$ of the variance in neopterin levels, whereas the eGFR was the strongest determinant of neopterin levels, explaining $53.7 \%$ of the variance in neopterin levels, in elective cardiac surgery patients with delirium. These findings may suggest that, in acutely ill medical patients, neopterin levels are mainly determined by inflammation/oxidative stress, whereas in elective cardiac surgery patients, neopterin levels are mainly driven by renal function/fluid status. However, it should be noticed that the majority of the medical patients was admitted with an infection.

In both groups of patients with delirium, the model based on age, CRP and eGFR explained approximately half of the variance in neopterin levels and therefore the remaining variance might be determined by other factors, eg, the severity 
A
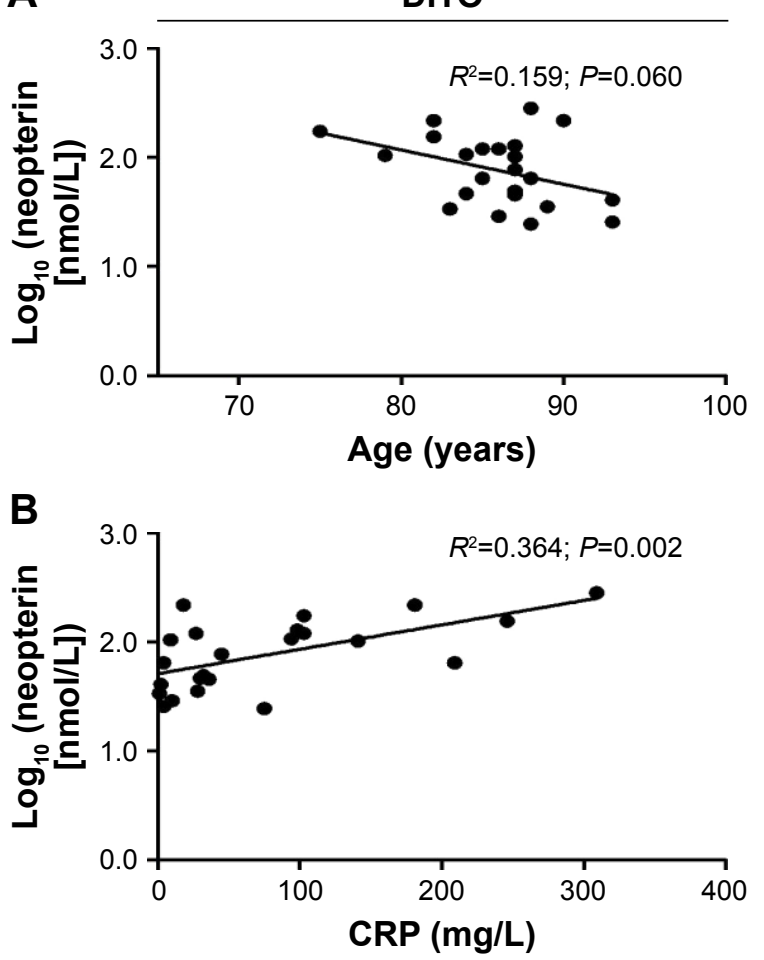

C
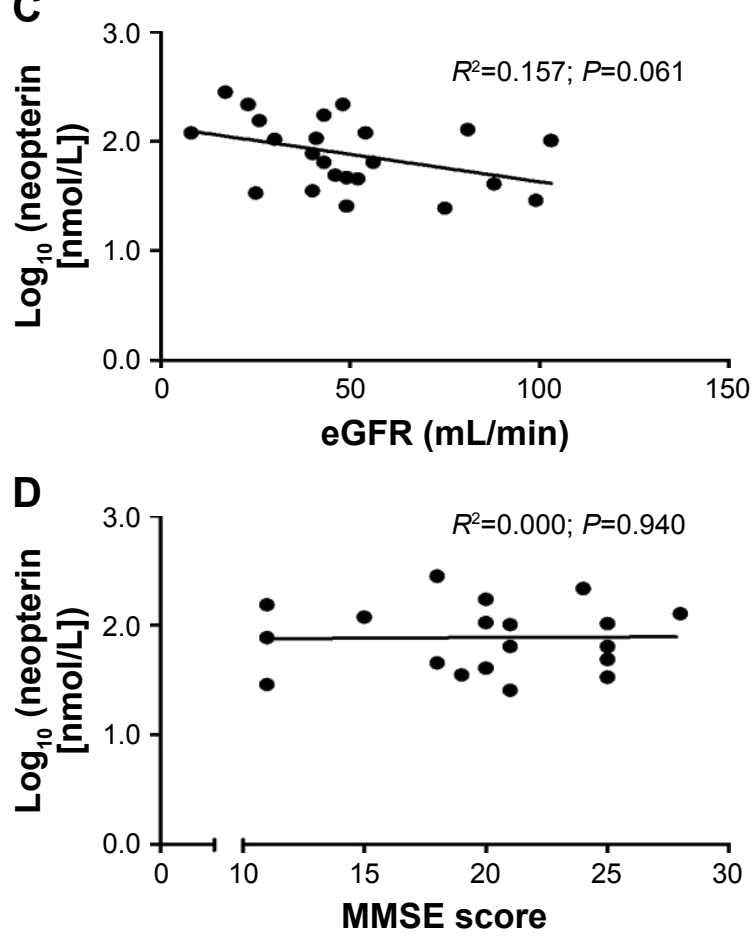
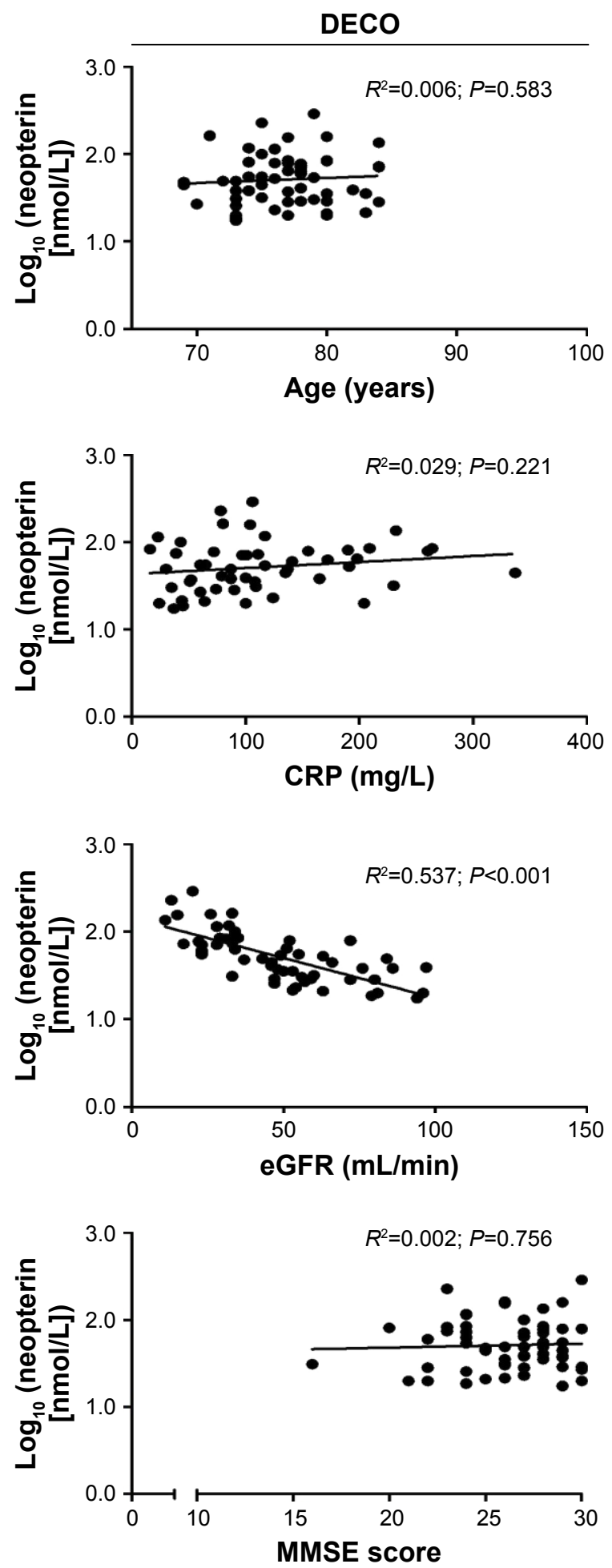

Figure I Scatterplots showing the relation between neopterin and age (A), CRP (B), eGFR (C) and MMSE score (D) in acutely ill medical patients with delirium (DITO) and patients with delirium after elective cardiac surgery (DECO).

Abbreviations: CRP, C-reactive protein; DITO, Delirium In The Old; DECO, DElirium pathogenesis, Cognition and Outcome; eGFR, estimated glomerular filtration rate; MMSE, Mini-Mental State Examination.

of delirium, ${ }^{11}$ the presence of comorbid cognitive disorders, other medical conditions ${ }^{8}$ or the use of specific drugs, such as aspirin ${ }^{30}$ and statins. ${ }^{31}$ Several studies have shown that a decrease in cognitive performance is accompanied by an increase in neopterin levels. ${ }^{32,33}$ In the present study, we found that acutely ill medical patients with delirium had significantly lower MMSE scores than patients who developed a delirium after elective cardiac surgery, but it should 
Table 4 Multiple linear regression analyses of potential determinants of neopterin

\begin{tabular}{|c|c|c|c|c|c|c|c|c|c|c|}
\hline \multirow[t]{2}{*}{ Variable } & \multicolumn{5}{|c|}{$\log _{10}($ neopterin $[\mathrm{nmol} / \mathrm{L}])$ in DITO } & \multicolumn{5}{|c|}{$\log _{10}($ neopterin $[\mathrm{nmol} / \mathrm{L}])$ in DECO } \\
\hline & B & $95 \% \mathrm{Cl}$ & $P$-value & $\mathbf{S r}^{2}$ & $\begin{array}{l}\text { Variance } \\
\text { explained, \% }\end{array}$ & B & $95 \% \mathrm{Cl}$ & $P$-value & $\mathrm{Sr}^{2}$ & $\begin{array}{l}\text { Variance } \\
\text { explained, \% }\end{array}$ \\
\hline Intercept & 3.923 & - & - & - & - & 2.336 & - & - & - & - \\
\hline Age & -0.024 & $(-0.05 I ; 0.003)$ & 0.078 & 0.085 & 8.53 & -0.003 & $(-0.018 ; 0.011)$ & 0.654 & 0.002 & 0.18 \\
\hline CRP & 0.002 & $(0.00 I ; 0.003)$ & 0.003 & 0.284 & 28.4 & 0.001 & $(0.000 ; 0.00 I)$ & 0.084 & 0.028 & 2.76 \\
\hline \multirow[t]{2}{*}{ eGFR } & -0.003 & $(-0.007 ; 0.002)$ & 0.221 & 0.040 & 3.96 & -0.009 & $(-0.012 ;-0.007)$ & 0.000 & 0.531 & 53.1 \\
\hline & \multicolumn{5}{|c|}{$R^{2}=0.532 ; F(3,19)=7.188 ; P=0.002$} & \multicolumn{5}{|c|}{$R^{2}=0.567 ; F(3,49)=21.4 I 2 ; P=0.000$} \\
\hline
\end{tabular}

Notes: Age was measured in years, CRP was measured in $\mathrm{mg} / \mathrm{L}$ and eGFR was measured in $\mathrm{mL} / \mathrm{min}$. B, regression coefficient; Sr${ }^{2}$, squared semi-partial correlation coefficient. Abbreviations: CRP, C-reactive protein; DITO, Delirium In The Old; DECO, DElirium pathogenesis, Cognition and Outcome; eGFR, estimated glomerular filtration rate.

be noted that in acutely ill medical patients MMSE scores were obtained after the delirium episode and therefore, the episode can have had residual effects on the MMSE score. Nevertheless, this finding might also suggest that acutely ill medical patients were cognitively more impaired than the elective cardiac surgery patients. However, when we added MMSE score as covariate to the analyses, estimates did not change and therefore, we are not able to confirm that the difference in mean neopterin levels is caused by a difference in cognitive performance between the two groups.

In the present study, we found no correlation between $\mathrm{CRP}$ and neopterin levels in elective cardiac surgery patients, although CRP levels were significantly higher in elective cardiac surgery patients than in acutely ill medical patients. A previous study, performed in patients who underwent CABG, showed that both CRP and neopterin levels increased after on-pump CABG, but the increase was significantly greater for CRP than for neopterin. ${ }^{34}$ This finding suggests a poor correlation between CRP and neopterin after on-pump CABG, which is in line with our findings. Since CRP is produced by other cells than neopterin, it might be possible that CRP levels reflect the activity of several other components of the immune system in elective cardiac surgery patients than in acutely ill medical patients.

$\mathrm{BH}_{4}$ functions as an essential cofactor in several enzymatic reactions involved in the production of serotonin and dopamine, ${ }^{20}$ two neurotransmitters that may play a role in delirium. ${ }^{7}$ The Phe/Tyr ratio is an indirect measure of the $\mathrm{BH}_{4}$ status, as it reflects the activity of the enzyme phenylalanine hydroxylase, an enzyme that uses $\mathrm{BH}_{4}$ as an essential cofactor. ${ }^{20,21}$ An elevated ratio of phenylalanine to tyrosine might be suggestive of a decreased $\mathrm{BH}_{4}$ availability. To the best our knowledge, $\mathrm{BH}_{4}$ status in relation to delirium has only been investigated in the DITO and DECO studies. In the DITO study, an increased Phe/Tyr ratio was found in acutely ill patients with delirium compared to those without, ${ }^{19}$ whereas in the DECO study, no differences were found in both the $\mathrm{BH}_{4}$ level and the Phe/Tyr ratio between patients who developed a delirium after elective cardiac surgery and those who did not. ${ }^{10}$ In the present study, we found higher Phe/Tyr ratios in acutely ill medical patients with delirium than in patients who developed a delirium after elective cardiac surgery. This finding might suggest a decreased availability of the essential cofactor $\mathrm{BH}_{4}$ in the production of serotonin and dopamine in acutely ill medical patients with delirium. No determinants of the Phe/Tyr ratio could be identified.

Although we found differences in the mean levels of neopterin and Phe/Tyr ratios and in the determinants of neopterin between the two groups of patients, the potential role of these markers in the pathophysiology of delirium needs to be elucidated in future studies. It remains speculative why mean neopterin levels in surgical patients with delirium are 1) lower than mean neopterin levels in acutely ill medical patients with delirium and 2) comparable with mean neopterin levels measured in acutely ill medical patients without delirium. ${ }^{9}$ This observation might suggest different threshold levels of neopterin in the development of delirium. Also, it cannot be excluded that factors other than the inflammatory response, eg, a low intraoperative perfusion pressure, hypoxia or changes in cerebral autoregulation, ${ }^{35,36}$ play a more dominant role in the pathogenesis of delirium after cardiac surgery. This hypothesis is in line with the results of a previous study in which the magnitude of the inflammatory response after cardiac surgery did not show a relationship with the development of postoperative cognitive dysfunction. ${ }^{37}$

This study has some limitations. First, the two studies used in the present study were not developed to compare these with each other. Inclusion and exclusion criteria were different and this might have influenced the results. Although we have adjusted our analyses for several covariates, we cannot exclude residual confounding. Second, considering 
the relatively small groups of patients with delirium, the findings need to be confirmed in a larger study. Third, due to the exploratory nature of this study, we did not adjust for multiple comparisons; therefore, we cannot exclude the possibility that the findings were obtained by chance. Using a Bonferroni correction for multiple comparisons, a $P$-value $<0.002$ would be required to denote statistical significance. In this case, the difference in Phe/Tyr ratio would be no longer statistically significant. Fourth, it might be possible that the degree of cognitive functioning has influenced our results. Although MMSE scores were obtained after delirium in acutely ill medical patients, the delirium episode can have had residual effects on the MMSE score. However, we have adjusted our statistical analyses for the CCI; therefore, we believe that we have provided an indirect adjustment for dementia. Fifth, the blood samples in the DITO and DECO studies were not analyzed at the same time and therefore, one might speculate that this can have introduced some bias. However, we do not think that this has influenced our results. In each HPLC run, several samples of the former run were reanalyzed to check for precision. If the values were within a range of 5\% of the results of the former analysis, the run was defined as good/accurate. Additionally, depending on the compound measured, all HPLC runs included external/internal standards and recovery measurements, as previously described. ${ }^{21,26,27}$ Sixth, we used CRP as indirect marker of inflammation in the analyses performed to identify possible determinants of neopterin. However, CRP is an acute phase reactant and might be a poor marker of cell-mediated immune system activation. Therefore, it might be possible that cell-mediated immune system activation and oxidative stress have played a larger role in both patient groups. Adjustment for inflammation markers other than CRP (eg, IFN- $\gamma$ ) would probably have given more insights in the results.

The present study has several strengths. First, the investigated markers were determined in the same laboratory using identical procedures; therefore, potential differences in mean levels of the investigated markers due to differences in the assay are unlikely. Second, in the two studies, patients were intensively monitored for clinical symptoms of delirium and the diagnosis of delirium was made by a geriatrician or psychiatrist using DSM-IV criteria. This makes it less likely that delirium was missed or that symptoms were misdiagnosed.

\section{Conclusion}

In this study, we found that acutely ill medical patients with delirium had higher levels of neopterin and higher Phe/Tyr ratios than patients who developed delirium after elective cardiac surgery. Differences in determinants of neopterin levels were found between the two groups. Our findings may suggest that, in acutely ill medical patients, neopterin levels are mainly determined by inflammation/oxidative stress whereas in elective cardiac surgery patients, neopterin levels are mainly driven by renal function/fluid status. These findings could suggest that the markers and pathways that might be involved in the pathophysiology of delirium in acutely ill medical patients may differ from those in elective cardiac surgery patients. Longitudinal studies with repeated measurements over time are needed to investigate whether the pathophysiology of delirium is different among groups. A major step forward in delirium research would be a large study, including several health care settings (eg, surgical, medical and intensive care units), in which potential biomarkers and other factors are compared across different groups of patients.

\section{Acknowledgments}

The DITO study was supported by a research grant of Fund NutsOhra. The DECO study was funded by the Department of Psychiatry, Erasmus MC, University Medical Center Rotterdam, the Netherlands.

\section{Disclosure}

The authors report no conflicts of interests in this work.

\section{References}

1. American Psychiatric Association. Diagnostic and Statistical Manual of Mental Disorders. 4th ed. Text revision. Washington, DC: American Psychiatric Association; 2000.

2. American Psychiatric Association. Diagnostic and Statistical Manual of Mental Disorders. 5th ed. Arlington, VA: American Psychiatric Association; 2013.

3. Inouye SK, Westendorp RG, Saczynski JS. Delirium in elderly people. Lancet. 2014;383(9920):911-922.

4. Witlox J, Eurelings LS, de Jonghe JF, Kalisvaart KJ, Eikelenboom P, van Gool WA. Delirium in elderly patients and the risk of postdischarge mortality, institutionalization, and dementia: a meta-analysis. JAMA 2010;304(4):443-451.

5. Lagarto L, Cerejeira J. Identification of sub-groups in acutely ill elderly patients with delirium: a cluster analysis. Int Psychogeriatr. 2016; 28(8):1283-1292.

6. van den Boogaard M, Kox M, Quinn KL, et al. Biomarkers associated with delirium in critically ill patients and their relation with long-term subjective cognitive dysfunction; indications for different pathways governing delirium in inflamed and noninflamed patients. Crit Care. 2011;15(6):R297.

7. Maldonado JR. Neuropathogenesis of delirium: review of current etiologic theories and common pathways. Am J Geriatr Psychiatry. 2013;21(12):1190-1222.

8. Berdowska A, Zwirska-Korczala K. Neopterin measurement in clinical diagnosis. J Clin Pharm Ther. 2001;26(5):319-329.

9. Egberts A, Wijnbeld EH, Fekkes D, et al. Neopterin: a potential biomarker for delirium in elderly patients. Dement Geriatr Cogn Disord. 2015;39(1-2):116-124. 
10. Osse RJ, Fekkes D, Tulen JH, et al. High preoperative plasma neopterin predicts delirium after cardiac surgery in older adults. $J$ Am Geriatr Soc. 2012;60(4):661-668.

11. Hall RJ, Watne LO, Idland AV, et al. Cerebrospinal fluid levels of neopterin are elevated in delirium after hip fracture. $J$ Neuroinflammation. 2016;13(1):170.

12. van der Mast RC, van den Broek WW, Fekkes D, Pepplinkhuizen L, Habbema JD. Is delirium after cardiac surgery related to plasma amino acids and physical condition? J Neuropsychiatry Clin Neurosci. 2000; 12(1):57-63.

13. van der Mast RC, Fekkes D, Moleman P, Pepplinkhuizen L. Is postoperative delirium related to reduced plasma tryptophan? Lancet. 1991; 338(8771):851-852.

14. Pandharipande PP, Morandi A, Adams JR, et al. Plasma tryptophan and tyrosine levels are independent risk factors for delirium in critically ill patients. Intensive Care Med. 2009;35(11):1886-1892.

15. Robinson TN, Raeburn CD, Angles EM, Moss M. Low tryptophan levels are associated with postoperative delirium in the elderly. Am J Surg. 2008;196(5):670-674.

16. van der Cammen TJ, Tiemeier H, Engelhart MJ, Fekkes D. Abnormal neurotransmitter metabolite levels in Alzheimer patients with a delirium. Int J Geriatr Psychiatry. 2006;21(9):838-843.

17. Flacker JM, Lipsitz LA. Large neutral amino acid changes and delirium in febrile elderly medical patients. J Gerontol A Biol Sci Med Sci. 2000;55(5):B249-B252; discussion B253-B244.

18. de Jonghe A, van Munster BC, Fekkes D, van Oosten HE, de Rooij SE. The tryptophan depletion theory in delirium: not confirmed in elderly hip fracture patients. Psychosomatics. 2012;53(3):236-243.

19. Egberts A, Fekkes D, Wijnbeld EH, et al. Disturbed serotonergic neurotransmission and oxidative stress in elderly patients with delirium. Dement Geriatr Cogn Dis Extra. 2015;5(3):450-458.

20. Werner ER, Blau N, Thöny B. Tetrahydrobiopterin: biochemistry and pathophysiology. Biochem J. 2011;438(3):397-414.

21. van Gool AR, Fekkes D, Kruit WH, et al. Serum amino acids, biopterin and neopterin during long-term immunotherapy with interferon-alpha in high-risk melanoma patients. Psychiatry Res. 2003;119(1-2): 125-132.

22. Schuurmans MJ, Shortridge-Baggett LM, Duursma SA. The delirium observation screening scale: a screening instrument for delirium. Res Theory Nurs Pract. 2003;17(1):31-50.

23. Folstein MF, Folstein SE, McHugh PR. "Mini-mental state". A practical method for grading the cognitive state of patients for the clinician. J Psychiatr Res. 1975;12(3):189-198.

24. Ely EW, Inouye SK, Bernard GR, et al. Delirium in mechanically ventilated patients: validity and reliability of the confusion assessment method for the intensive care unit (CAM-ICU). JAMA. 2001;286(21): 2703-2710.
25. Laich A, Neurauter G, Wirleitner B, Fuchs D. Degradation of serum neopterin during daylight exposure. Clin Chim Acta. 2002;322(1-2): 175-178.

26. Fekkes D. Automated analysis of primary amino acids in plasma by high-performance liquid chromatography. Methods Mol Biol. 2012; 828:183-200.

27. Fekkes D, Timmerman L, Pepplinkhuizen L. Effects of clomipramine on plasma amino acids and serotonergic parameters in panic disorder and depression. Eur Neuropsychopharmacol. 1997;7(3):235-239.

28. de Rooij SE, van Munster BC, Korevaar JC, Levi M. Cytokines and acute phase response in delirium. J Psychosom Res. 2007;62(5):521-525.

29. Kazmierski J, Banys A, Latek J, Bourke J, Jaszewski R. Raised IL-2 and TNF- $\alpha$ concentrations are associated with postoperative delirium in patients undergoing coronary-artery bypass graft surgery. Int Psychogeriatr. 2014;26(5):845-855.

30. Egberts A, Fekkes D, Ziere G, van der Cammen T, Mattace-Raso F. Potential influence of aspirin on neopterin and tryptophan levels in patients with a delirium. Geriatrics. 2016;1(2):10.

31. Avanzas P, Arroyo-Espliguero R, Quiles J, Roy D, Kaski JC. Elevated serum neopterin predicts future adverse cardiac events in patients with chronic stable angina pectoris. Eur Heart J. 2005;26(5):457-463.

32. Parker DC, Mielke MM, Yu Q, et al. Plasma neopterin level as a marker of peripheral immune activation in amnestic mild cognitive impairment and Alzheimer's disease. Int J Geriatr Psychiatry. 2013;28(2):149-154.

33. Blasko I, Knaus G, Weiss E, et al. Cognitive deterioration in Alzheimer's disease is accompanied by increase of plasma neopterin. J Psychiatr Res. 2007;41(8):694-701.

34. Ayaz L, Unlu A, Sucu N, Tamer L, Atik U, Sungur MA. Role of neopterin, C-reactive protein and myeloperoxidase in patients undergoing cardiopulmonary bypass. Med Princ Pract. 2010;19(6):479-484.

35. Siepe M, Pfeiffer T, Gieringer A, et al. Increased systemic perfusion pressure during cardiopulmonary bypass is associated with less early postoperative cognitive dysfunction and delirium. Eur J Cardiothorac Surg. 2011;40(1):200-207.

36. Hollinger A, Siegemund M, Goettel N, Steiner LA. Postoperative delirium in cardiac surgery: an unavoidable menace? J Cardiothorac Vasc Anesth. 2015;29(6):1677-1687.

37. Nemeth E, Vig K, Racz K, et al. Influence of the postoperative inflammatory response on cognitive decline in elderly patients undergoing on-pump cardiac surgery: a controlled, prospective observational study. BMC Anesthesiol. 2017;17(1):113. 


\section{Supplementary material}

Table SI Mean levels of biochemical parameters after additional adjustment for MMSE score

\begin{tabular}{|c|c|c|c|}
\hline & DITO $(n=19)^{a}$ & $\operatorname{DECO}(n=57)$ & $P$-value \\
\hline Neopterin, nmol/L & $97.9(68.4-140.3)$ & $47.3(39.9-56.0)$ & $0.002^{\mathrm{b}}$ \\
\hline Homovanillic acid, nmol/L & $111.2(65.9-187.9)^{c}$ & $162.6(129.1-204.6)^{c}$ & 0.252 \\
\hline Glutamic acid, $\mu \mathrm{mol} / \mathrm{L}$ & $42.2(31.9-55.8)$ & $36.7(32.4-41.7)$ & 0.436 \\
\hline Serine, $\mu \mathrm{mol} / \mathrm{L}$ & $76.6(63.0-93.3)$ & $76.6(70.0-83.8)$ & 0.994 \\
\hline Glycine, $\mu \mathrm{mol} / \mathrm{L}$ & I79.| (|49.6-2|4.3) & $198.2(|82.8-2| 4.8)$ & 0.376 \\
\hline Citrulline, $\mu \mathrm{mol} / \mathrm{L}$ & $20.7(\mid 15.9-26.9)$ & $24.2(21.5-27.2)$ & 0.349 \\
\hline Arginine, $\mu \mathrm{mol} / \mathrm{L}$ & $35.1(26.2-47.0)$ & $44.5(38.9-50.8)$ & 0.204 \\
\hline Taurine, $\mu \mathrm{mol} / \mathrm{L}$ & $33.7(23.8-47.8)$ & $33.6(29.2-40.1)$ & 0.946 \\
\hline Tyrosine, $\mu \mathrm{mol} / \mathrm{L}$ & $54.3(43.8-67.5)$ & $67.0(60.7-74.0)$ & 0.130 \\
\hline Valine, $\mu \mathrm{mol} / \mathrm{L}$ & $205.6(174.2-242.1)$ & $204.2(|89.2-2| 9.8)$ & 0.950 \\
\hline Methionine, $\mu \mathrm{mol} / \mathrm{L}$ & $21.7(17.1-27.6)$ & $28.4(25.5-31.7)$ & 0.079 \\
\hline Tryptophan, $\mu \mathrm{mol} / \mathrm{L}^{\mathrm{d}}$ & $32.0(23.4-40.7)$ & $33.5(29.6-37.4)$ & 0.787 \\
\hline Phenylalanine, $\mu \mathrm{mol} / \mathrm{L}$ & $78.0(67.9-89.3)$ & $76.6(7|.9-8| .5)$ & 0.837 \\
\hline Isoleucine, $\mu \mathrm{mol} / \mathrm{L}$ & $56.9(46.5-69.5)$ & $67.5(61.5-74.0)$ & 0.183 \\
\hline Leucine, $\mu \mathrm{mol} / \mathrm{L}$ & I2I.I (99.8-147.2) & I $24.7(|| 4.3-136.1)$ & 0.816 \\
\hline Phenylalanine/tyrosine ratio & $1.44(1.21-1.69)$ & $1.14(1.06-1.23)$ & 0.035 \\
\hline Tryptophan/LNAAs ratio $\times 100^{d}$ & $5.63(4.40-6.86)$ & $6.06(5.51-6.62)$ & 0.576 \\
\hline Tyrosine/LNAAs ratio $\times 100$ & $10.9(9.2-13.1)$ & I3.2(I2.2-14.3) & 0.104 \\
\hline Phenylalanine/LNAAs ratio $\times 100$ & I6.5 (14.3-19.1) & I5.3 (14.4-16.4) & 0.438 \\
\hline Citrulline/arginine ratio & $0.59(0.43-0.82)$ & $0.54(0.47-0.63)$ & 0.684 \\
\hline
\end{tabular}

Notes: Values are expressed as mean $(95 \% \mathrm{Cl})$ and are the back-transformed $\log _{10}$ values unless otherwise specified. Models are adjusted for age, sex, CCl and MMSE score. The model including neopterin is adjusted for age, sex, CCl, the eGFR, CRP and MMSE score. aAnalyses were performed in 19 patients instead of 23 patients due to

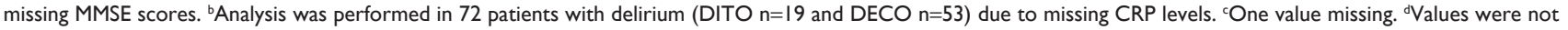
logarithmically transformed.

Abbreviations: CCI, Charlson Comorbidity Index; CRP, C-reactive protein; eGFR, estimated glomerular filtration rate; LNAAs, large neutral amino acids; MMSE, MiniMental State Examination.

Clinical Interventions in Aging

\section{Publish your work in this journal}

Clinical Interventions in Aging is an international, peer-reviewed journal focusing on evidence-based reports on the value or lack thereof of treatments intended to prevent or delay the onset of maladaptive correlates of aging in human beings. This journal is indexed on PubMed Central, MedLine,

\section{Dovepress}

CAS, Scopus and the Elsevier Bibliographic databases. The manuscript management system is completely online and includes a very quick and fair peer-review system, which is all easy to use. Visit http://www.dovepress. com/testimonials.php to read real quotes from published authors. 